$63^{\text {ème }}$ Congrès de la SFCO, 03031 (2015)

DOI:10.1051/sfco/20156303031

(C) Owned by the authors, published by EDP Sciences, 2015

\title{
Thrombophlébite faciale superficielle non odontogène : à propos d'un cas
}

\author{
Doustkam A-A*, Gros C-I**, Montaner S*, Bornert F* \\ * Unité de Chirurgie Buccale-Implantologie \\ ** Unité de Radiologie dento-maxillaire, Pôle de Médecine et Chirurgie Bucco-Dentaires, Hôpital Civil, CHRU \\ de Strasbourg
}

Le diagnostic dethrombophlébite de la veine faciale est avant toutclinique. Celle-ci se traduit par un oedème du pli nasogénien diffusant jusqu'à l'angle interne de l'oeil. La palpation retrouve classiquement un cordon induré et l'évolution se fait vers le développement d'une tuméfaction douloureuse du canthus interne. La réaction inflammatoire persiste 2 à 3 semaines, l'induration palpable persiste beaucoup plus longtemps (Dagain et al. 2008).

Le cas d'une femme de 54 ans est rapporté. La patiente présente comme antécédents notables deux accidents vasculaires cérébraux, une bi-greffe cardio-pulmonaire et un déficit en facteur XII. Elle nous est adresséepar le service de pneumologie suite à la découverte fortuite d'une tuméfaction jugale haute, spontanément douloureuse, évoluant sur un mode aigu. La demande visait initialement à rechercher une origine dentaire par un examen CBCT dento-facial dans le cadre d'un diagnostic clinique de cellulite faciale.

En complément de la réalisation du CBCT, un examen clinique a été réalisé directement au service de radiologie par le praticien référent-CBCT à ce moment-là. Au niveau exobuccal, était noté une tuméfaction hémi-maxillaire gauche, avec palpation d'un cordon induré et douloureux au niveau du sillon naso-génien jusqu'au canthus interne associé à un œdème palpébral supérieur. Il n’y avait pas de point d'entrée cutané identifié. Au niveau endobuccal, la patiente était édentée totale, sans tuméfaction vestibulaire. Au final, le résultat CBCT était en faveur d'un comblement des cellules ethmoïdales compatible avec une ethmoïdite aiguë gauche et exclusion de foyers infectieux dentaires.

Face à ce tableau clinique, le diagnostic initial a été révisé en thrombophlébite de la veine faciale gauche conduisant à faire réaliser un bilan par un premier scanner cérébral complété ensuite par une angio-IRM. Une thrombose veineuse superficielle de la veine faciale gauche a été confirmée sans signes d'extension endocranienne.

Un traitement médicamenteux a été introduit par l'équipe médicale comprenant un antibiotique permettant une régression de l'ethmoïdite ainsi qu'un anti-thrombotique empêchant une complication sévère à savoir l'extension au sinus caverneux.

Ce cas a été l'occasion de rappeler une complication rare des foyers infectieux maxillo-faciaux depuis l'avènement des antibiotiques, mais nécessitant une détection précoce par une sémiologie clinique fine visant à diminuer la morbidité.

Références

A. Dagain, G. Thiéry, R. Dulou, J.-M. Delmas, P. Pernot. Thrombophlébites craniofaciales. EMC Médecine buccale 2008:1-12 [Article 28-355-B-10].

This is an Open Access article distributed under the terms of the Creative Commons Attribution License 4.0, which permits unrestricted use, distribution, and reproduction in any medium, provided the original work is properly cited. 
$63^{\text {ème }}$ Congrès de la SFCO

Nom et adresse du conférencier

Amir-Andre DOUSTKAM

UF de Chirurgie Buccale-Implantologie, Pôle de médecine et chirurgie bucco-dentaires, Hôpital Civil, CHRU de Strasbourg

1 place de l'hôpital

67000 Strasbourg (France)

amirandre.doustkam@chru-strasbourg.fr 